\title{
Article \\ Liensinine Prevents Vascular Inflammation by Attenuating Inflammatory Mediators and Modulating VSMC Function
}

\author{
Moon Young Jun ${ }^{1}$, Rajendra Karki ${ }^{1}$, Keshav Raj Paudel ${ }^{1} @$, Nisha Panth ${ }^{2}$, Hari Prasad Devkota ${ }^{3}($ and \\ Dong-Wook Kim ${ }^{1, *}$ \\ 1 Department of Oriental Medicine Resources, Mokpo National University, Muan-gun, Jeonnam 534-729, Korea; \\ herb1999@mokpo.ac.kr (M.Y.J.); Rajendra.Karki@stjude.org (R.K.); keshavraj.paudel@uts.edu.au (K.R.P.) \\ 2 College of Pharmacy and Natural Medicine Research Institute, Mokpo National University, Muan-gun, \\ Jeonnam 58554, Korea; n.panth@centenary.org.au \\ 3 Graduate School of Pharmaceutical Sciences, Kumamoto University, 5-1 Oe-honmachi, Chou-ku, \\ Kumamoto City, Kumamoto 862-0973, Japan; devkotah@kumamoto-u.ac.jp \\ * Correspondence: dbkim@mokpo.ac.kr
}

Citation: Jun, M.Y.; Karki, R.; Paudel, K.R.; Panth, N.;

Devkota, H.P.; Kim, D.-W. Liensinine Prevents Vascular Inflammation by Attenuating Inflammatory Mediators and Modulating VSMC Function. Appl. Sci. 2021, 11, 386. https://doi.org/10.3390/app11010386

Received: 13 December 2020 Accepted: 30 December 2020 Published: 3 January 2021

Publisher's Note: MDPI stays neutral with regard to jurisdictional clai$\mathrm{ms}$ in published maps and institutional affiliations.

Copyright: $@ 2021$ by the authors. Licensee MDPI, Basel, Switzerland. This article is an open access article distributed under the terms and conditions of the Creative Commons Attribution (CC BY) license (https:// creativecommons.org/licenses/by/ $4.0 /)$.

\begin{abstract}
Liensinine is a bisbenzylisoquinoline alkaloid found in various parts of the lotus (Nelumbo nucifera Gaertn.) including seeds. In this study, we explored the preventive activity of liensinine on vascular inflammation via attenuation of inflammatory mediators in macrophage and targeting the proliferation and migration of human vascular smooth muscle cells (VSMC). Anti-oxidative activity was evaluated by using the 1,1-diphenyl-2-picrylhydrazyl (DPPH) free radical scavenging assay method and measuring the peroxidation of serum lipid. Inflammatory markers were studied by evaluating the release of nitric oxide $(\mathrm{NO})$ and the protein levels of inducible nitric oxide synthase (iNOS) and cyclooxygenase (COX-2) in macrophage cells (RAW264.7) and interleukin (IL)-6 production in VSMC. Similarly, anti-proliferative activity in VSMC was evaluated by 3-(4,5-dimethylthiazol-2-yl)-2,5-diphenyltetrazolium bromide (MTT) assay. The enzymatic activity of matrix metalloproteinase (MMP)-9 in VSMC was evaluated by gelatin zymography. Liensinine possesses significant anti-oxidative activity as revealed by the DPPH assay and inhibition of serum lipid peroxidation. Likewise, liensinine decreased NO generation in RAW 264.7 cells. In VSMC, liensinine suppressed platelet-derived growth factor stimulated proliferation and tumor necrosis factor- $\alpha$ (TNF- $\alpha$ ) induced MMP-9 enzymatic activity as well as IL-6 expression. Our results revealed the potential preventive effect of liensinine on vascular inflammation, suggesting it as a promising compound for the prevention of vascular inflammation.
\end{abstract}

Keywords: vascular inflammation; liensinine; VSMC; macrophage; proliferation; migration

\section{Introduction}

Atherosclerosis is defined as a chronic vascular inflammatory disorder that progresses with the lipid oxidation due to hypercholesteremia, diabetes mellitus, hypertension and various other disorders [1]. Oxidized lipids induce the secretion of various cytokines and recruit macrophages and T-lymphocytes at the site of a lesion [2]. Further, accelerated vascular smooth muscle cell (VSMC) migration and proliferation contribute to atherosclerotic plaque development [3,4]. It is also stimulated by oxidative stress, which produces different inflammatory cytokines; tumor necrosis factor-a(TNF-a), interleukin-6 (IL-6) and growth factor such as platelet-derived growth factor-BB (PDGF-BB). According to the previous study, treatment of IL-6 to C57Bl/ 6 mice increased fatty streak cores by approximately five times as revealed by oil red o staining of aortic sinus serial section, and increased the release of inflammatory cytokine, IL-1 $\beta$ and TNF-ain the plasma [5]. Moreover, TNF-aand PDGF-BB are already reported to stimulate the migration of human aortic VSMC from media to the intima of blood vessels [6]. These migrated cells are extensively proliferated under the influence of inducing agents like PDGF, TNF-aand lipopolysaccharide (LPS) in 
the intimal layer of arteries forming atheroma [7-9]. Mitogen-activated protein kinases (MAPKs), a family of serine-threonine kinases, regulate cell adhesion, migration and proliferation on human aortic VSMC in response to external stimuli including TNF-a [10] and PDGF-BB [11,12]. The role of matrix metalloproteinase (MMP) is well known due to their function for disrupting matrices composed of gelatin or elastin, which could permit human aortic VSMC migration by destroying the elastic lamina present between the intima and media $[13,14]$. Vascular inflammation is a result of toxic insult by the mediators released by the macrophage. Initially, monocytes normally circulating in the blood vessel are migrated to tunica media due to endothelial dysfunction. At this site, they engulf the oxidized lowdensity lipoprotein (ox-LDL) and become activated after changing the morphology from macrophage to foam cell, as recognized by the accumulation of fatty streaks on lipid laden molecules $[15,16]$. Activated macrophages release inflammatory mediators like nitric oxide (NO) via the inducible nitric oxidase pathway, and prostaglandins via cyclooxygenase pathway. Collectively, these endogenous inflammatory agents trigger the formation of a necrotic core at the site of an atherosclerosis lesion $[17,18]$.

Liensinine is a bisbenzylisoquinoline alkaloid found in various part of the lotus (Nelumbo nucifera Gaertn.) including seeds (Figure 1). Liensinine and other bisbenzylisoquinoline alkaloids present in lotuses are reported as potent anticancer, anti-inflammatory, antioxidant, cardiovascular protective and neuroprotective agents [19-21]. Traditionally, the seed embryo of the lotus has been used as medicine in China for cardiovascular diseases, nervous disorders and sleeplessness [22]. Previously, we reported the anti-atherosclerotic activity of Nelumbo nucifera leaf extract and its alkaloid rich fraction through inhibition of neointimal hyperplasia in rats and inhibiting VCMC proliferation and migration [12,23]. In the current study, we aim to determine the similarly beneficial activity of the liensinine against vascular inflammation through anti-inflammatory, anti-proliferative, anti-migratory and anti-oxidative activities.

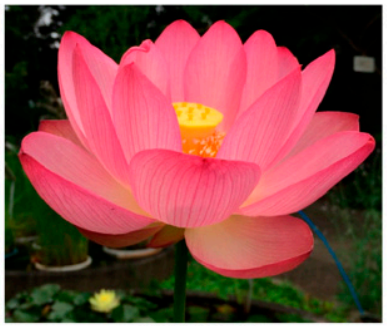

Flower

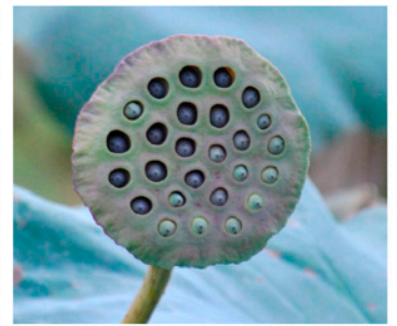

Seeds

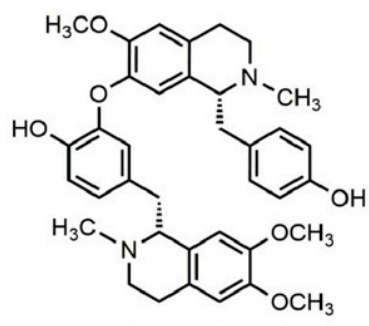

Liensinine

Figure 1. The flower and seeds of Nelumbo nucifera and the chemical structure of liensinine.

\section{Materials and Methods}

\subsection{Reagents}

Liensinine (Cas number: 2586-96-1) was purchased from Sigma Aldrich. 3-(4,5Dimethylthiazol-2-yl)-2,5-diphenyltetrazolium bromide (MTT) and lipopolysaccharide (LPS) were obtained from Sigma Aldrich (St Louis, MO, USA). PDGF-BB and TNF- $\alpha$ were purchased from R \& D systems (Minneapolis, MN, USA). All remaining common laboratory chemical reagents or solvents were purchased from Sigma-Aldrich, South Korea.

\subsection{Cell Culture}

VSMC from human aorta obtained from ATCC, USA and RAW264.7 cells purchased from Korean cell line bank (Seoul, Korea) were cultured in complete cell culture media with Dulbecco's modified Eagle's medium (DMEM), 10\% fetal bovine serum (FBS), and 1\% antibiotics (penicillin + streptomycin) in a standard cell incubator with $5 \% \mathrm{CO}_{2}$. The cells were incubated in media with $0.1 \%$ FBS for $24 \mathrm{~h}$ to allow them to synchronize at G0 phase for each assay. Liensinine was solubilized in dimethyl sulfoxide (DMSO) and diluted in a serum-free medium for treatment of cells. The final \% of DMSO while treating cells were below $0.1 \%$. 


\subsection{DPPH Assay and Thiobarbituric Acid Reactive Substance (TBARS) Assay for Lipid Peroxidation Assay}

The anti-oxidant activity of liensinine was evaluated using a DPPH free radical scavenging assay and measurement of serum lipid peroxidation was carried out using TBARS assay following the methods as described previously [11].

\subsection{Proliferation Assay}

PDGF-BB was used as a proliferation inducer in VSMC and \% proliferation was measured by MTT colorimetric assay as described previously [24]. VSMC were treated with liensinine $1 \mathrm{~h}$ before PDGF-BB and incubated for $24 \mathrm{~h}$ to allow proliferation. Freshly prepared MTT in phosphate buffer saline was added and incubated for an additional $4 \mathrm{~h}$. The purple color formazan developed due to the reduction of MTT by viable VSMC were dissolved with DMSO. Then, a colorimetric reading was taken by measuring absorbance at $540 \mathrm{~nm}$ with a microplate reader. The anti-proliferative effect of liensinine was evaluated by comparison with the control group (treated with PDGF-BB) as $100 \%$.

\subsection{Gelatin Zymography}

Gelatin zymography was carried out to examine the enzymatic activity of MMP-9 as described previously [14]. Briefly, VSMC were seeded in $60 \mathrm{~mm}$ petri plates at the density of $1 \times 10^{6}$ cells. Liensinine was added at a predetermined concentration for $1 \mathrm{~h}$ and cells were treated with TNF- $\alpha(100 \mathrm{ng} / \mathrm{mL})$ for the next $24 \mathrm{~h}$. The supernatant cell culture media was collected and $30 \mu \mathrm{g}$ of protein equivalent was used for electrophoresis in 10\% SDS-PAGE with $0.25 \%$ gelatin. Next, the gels were incubated in renaturating buffer $(2.5 \%$ Triton X-100) for half-an-hour and incubated again in developing buffer at $37^{\circ} \mathrm{C}$ for $16-24 \mathrm{~h}$. In order to visualize the bands of MMP2 and MMP-9, gels were stained with $0.05 \%$ Coomassie Brilliant Blue followed by incubation in destaining buffer. Photographs of the gel were taken to observe the proteolysis of gelatin by MMP-2 and MMP-9.

\subsection{Determination of IL-6 Release in TNF- $\alpha$ Stimulated VSMC}

VSMC was pretreated with $1-30 \mu \mathrm{M}$ of liensinine for $1 \mathrm{~h}$ and further treated with TNF$\alpha$ for the next $24 \mathrm{~h}$. The level of IL- 6 released by cells was measured in culture supernatant using an ELISA kit of IL-6, according to manufacturer's protocol.

\subsection{Cell Viability/Cytotoxicity Assay, NO Release and Immunoblot of iNOS, and COX-2 Protein} Expression in RAW264.7 Cells

RAW264.7 cell viability or cytotoxicity assay was carried out using the MTT colorimetric assay as described in Section 2.4 (without any stimulant). The NO levels in RAW264.7 cells were evaluated as mentioned previously [25]. Briefly, the cells were treated with $1-20 \mu \mathrm{M}$ of liensinine for $1 \mathrm{~h}$, then induced with LPS at $1 \mu \mathrm{g} / \mathrm{mL}$ for the next $24 \mathrm{~h}$. The level of NO in culture supernatant was measured by mixing a 1:1 ratio $(100 \mu \mathrm{L})$ of supernatant: Griess reagent. The colored product was measured calorimetrically by reading absorbance at $540 \mathrm{~nm}$. For protein expression of iNOS and COX-2 in RAW 264.7 cells, immunoblotting was carried out [26].

\subsection{Statistical Analysis}

Data analysis and graphs were prepared using SigmaPlot or Microsoft Excel. The data are represented as mean \pm standard error mean. Multiple groups were compared using one-way analysis of variance (ANOVA) and Duncan's post-hoc test. $p$-values of $<0.05$ were considered as statistically significant.

\section{Results}

\subsection{Liensinine Scavenges DPPH Free Radicals and Inhibits Serum Lipid Peroxidation}

Liensinine showed concentration-dependent DPPH free radical scavenging activity as displayed in Figure 2a. The inhibitory concentration $50\left(\mathrm{IC}_{50}\right)$ of liensinine was found 
to be $1.8 \mu \mathrm{g} / \mathrm{mL}$. Figure $2 \mathrm{~b}$ shows the measurement of serum lipid peroxidation. Liensinine at concentrations of 30 and $40 \mu \mathrm{g} / \mathrm{mL}$ showed remarkable reduction of serum lipid peroxidation in terms of TBARS value.

(a)

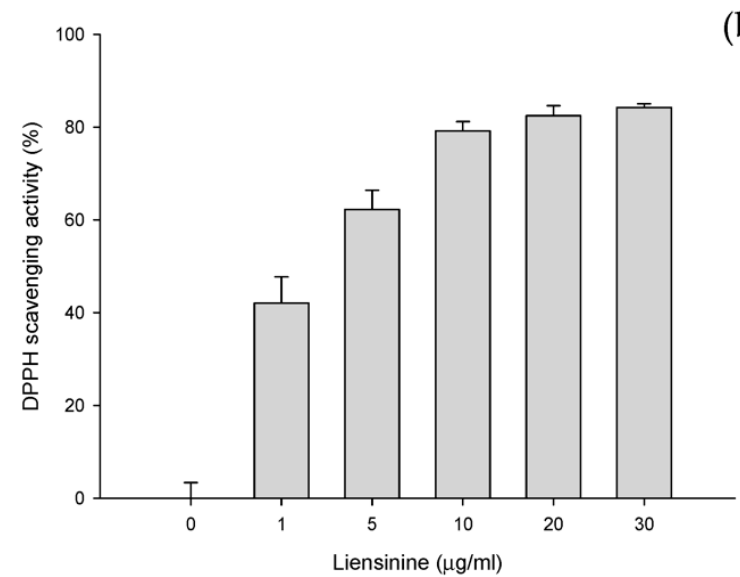

(b)

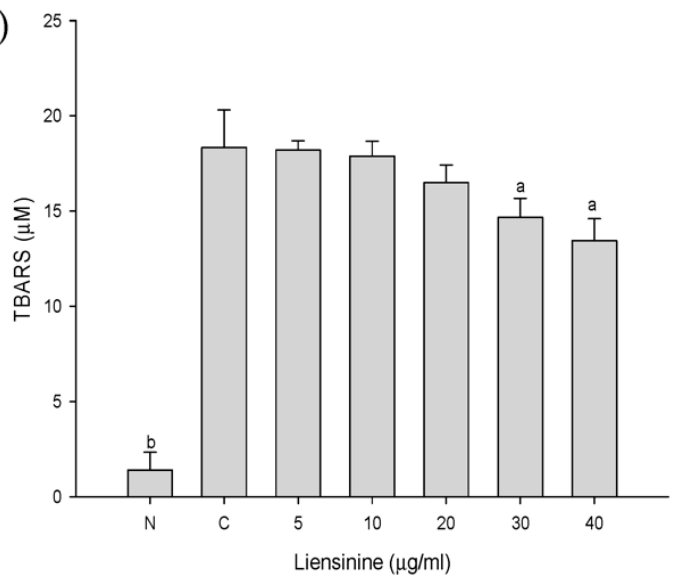

Figure 2. The antioxidant potency of liensinine. (a) 1,1-diphenyl-2-picrylhydrazyl (DPPH) free radical scavenging activity $\left(\mathrm{IC}_{50}=1.8 \mu \mathrm{g} / \mathrm{mL}\right.$ and $\left.\mathrm{r}^{2}=0.94\right)(\mathrm{b})$ Serum lipid peroxidation inhibitory activity. $\mathrm{N}$ represents the normal group (without copper sulphate). ${ }^{\mathrm{a}} p<0.05$ and ${ }^{\mathrm{b}} p<0.01$ vs. $\mathrm{C}$ (the control group; presence of copper sulfate only). $\mathrm{n}=3$ replicates.

\subsection{Liensinine Inhibits VSMC Proliferation}

PDGF-BB is a potent growth factor over-expressed in human coronary arteries during atherosclerosis and restenosis [27]. Figure 3 shows the potent anti-proliferative activity of liensinine against PDGF-BB stimulated VSMC proliferation. Liensinine, at a concentrations of 20 and $30 \mu \mathrm{g} / \mathrm{mL}$ notably decreased the \% cell proliferation to $67.16 \%$ and $47.02 \%$, respectively, vs. $100 \%$ of control (PDGF-BB only).

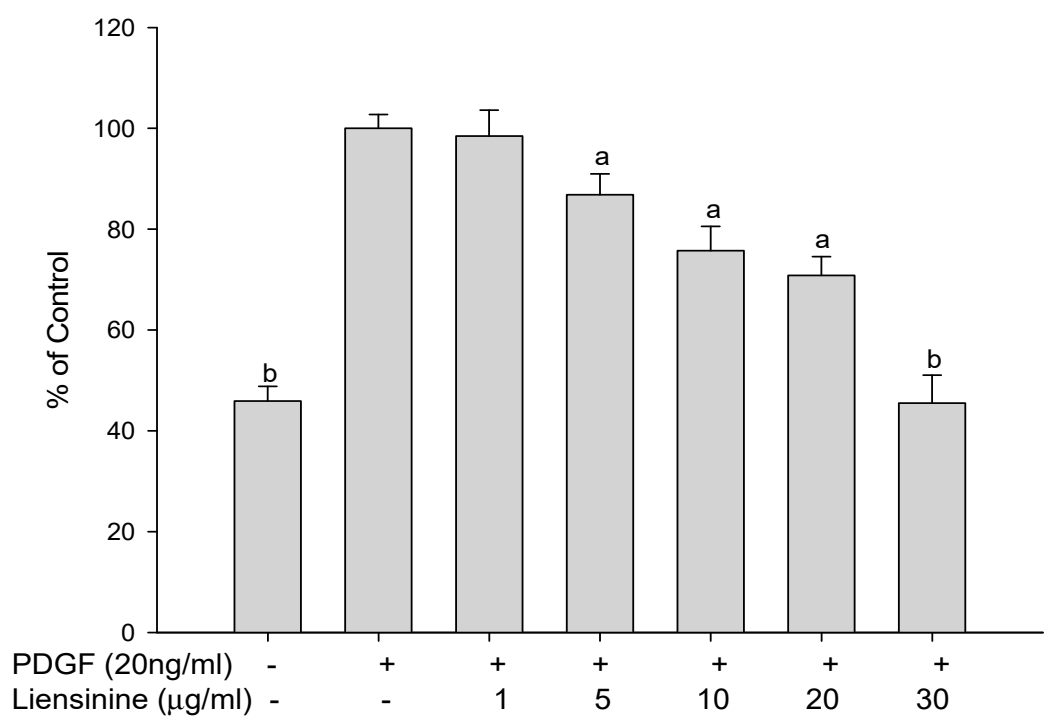

Figure 3. The effect of liensinine on platelet-derived growth factor-BB (PDGF-BB) induced proliferation of human vascular smooth muscle cells (VSMC). VSMC were pretreated with different concentration of liensinine for $1 \mathrm{~h}$ followed by stimulation with $20 \mathrm{ng} / \mathrm{mL}$ of PDGF-BB for $24 \mathrm{~h}$. The effect on proliferation of VSMC was evaluated by MTT assay. ${ }^{\mathrm{a}} p<0.05$ and ${ }^{\mathrm{b}} p<0.01$ vs. Control (only PDGF). $\mathrm{n}=3$ replicates.

\subsection{Liensinine Inhibits MMP-9 Enzymatic Action}

The degradation of the extracellular matrix by enzymatic action of MMPs (stimulated by various mitogens) is responsible for VSMC migration. TNF- $\alpha$ is one such mi- 
togen/activator that stimulates MMP-9 enzyme activity in VSMCs [28]. The effect of liensinine on the MMP-9 proteolytic degradation of gelatin in VSMCs is displayed in Figure 4. The MMP-9 band was drastically increased by TNF- $\alpha$ (compared to without TNF- $\alpha$ ) and decreased concentration dependently by liensinine (compared to TNF- $\alpha$ ).

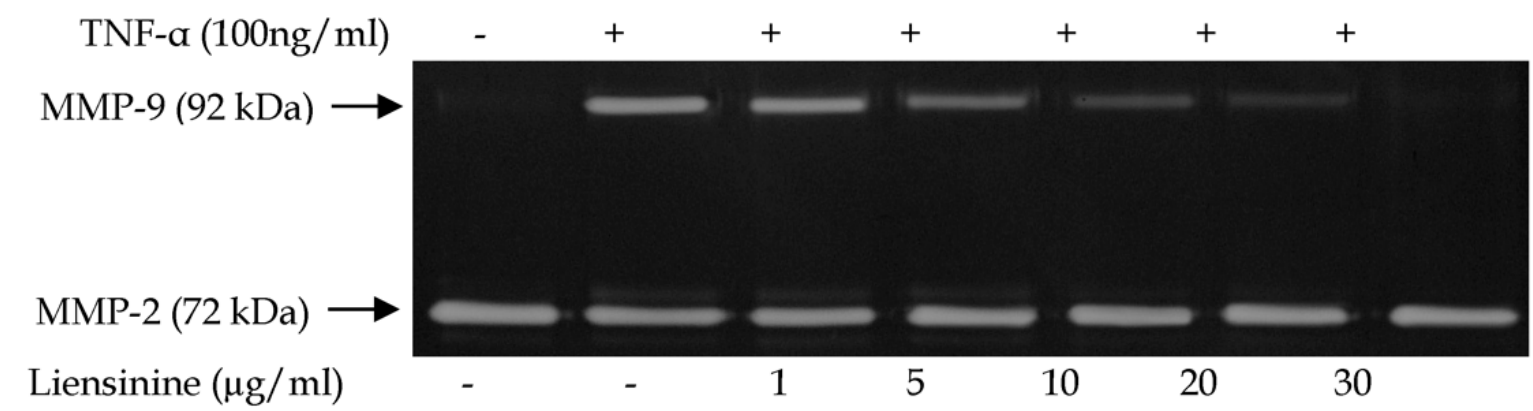

Figure 4. The effect of liensinine on matrix metalloproteinase (MMPs) secretion in TNF- $\alpha$ stimulated VSMC. VSMC were pretreated with different concentrations of liensinine and stimulated with TNF- $\alpha$. The effect of liensinine on MMPs proteolytic/enzymatic activity was observed by gelatin zymography. The photographs of the gel were taken after Coomassie Brilliant Blue staining. $\mathrm{n}=3$ replicates.

\subsection{Liensinine Inhibits IL-6 in VSMC}

As shown in Figure 5, TNF- $\alpha$ significantly increased the IL-6 production in VSMC by 3 -fold compared to the control (without TNF- $\alpha$ ) while liensinine at a concentration of 10 , $20,30 \mu \mathrm{g} / \mathrm{mL}$ significantly inhibited the IL-6 release.

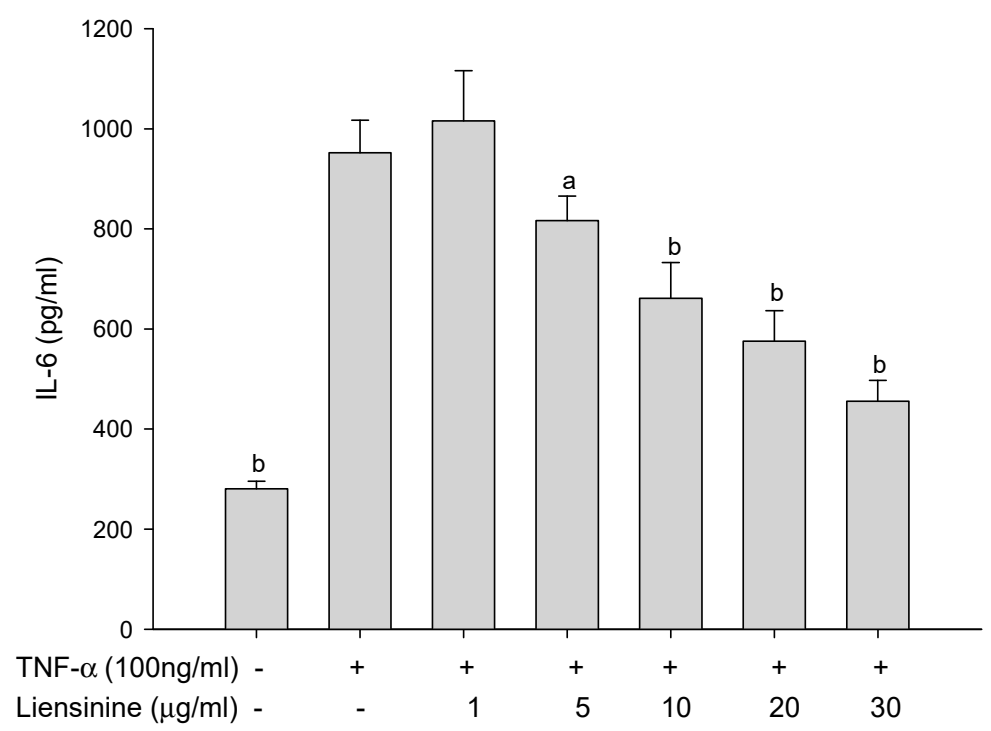

Figure 5. The effect of liensinine in IL-6 release in TNF- $\alpha$ stimulated VSMC. VSMC were pretreated with different concentrations of liensinine and stimulated with TNF- $\alpha$. The effect of liensinine on IL-6 release was measured by IL-6 ELISA Kit. Liensinine concentration-dependently inhibited the IL-6 release in VSMC. ${ }^{\mathrm{a}} p<0.05$ and $^{\mathrm{b}} p<0.01$ vs. Control (only TNF- $\alpha$ ). $\mathrm{n}=3$ replicates.

\subsection{Liensinine Suppresses NO Production and Inhibit Protein Expression of iNOS and COX-2} in RAW264.7

First, we checked the cytotoxicity of various concentrations of liensinine in RAW264.7 cells. Liensinine up to $20 \mu \mathrm{M}$ did not exert any significant decrease in RAW264.7 cell viability (Figure 6a). The $24 \mathrm{~h}$ treatment of cells with LPS increased the production of NO $(95.2 \pm 9.8 \mu \mathrm{M})$ by approximately 3-fold compared to those without LPS treatment. Liensinine showed inhibition of NO release in a concentration-dependent manner (Figure 6b). 
Consistent with NO production, there was an overexpression of iNOS (Figure $6 c, d$ ) and COX-2 (Figure 6c,e) proteins after LPS treatment, while liensinine (5-20 $\mu \mathrm{M})$ treatment resulted in a notable reduction in protein expression.

(a)

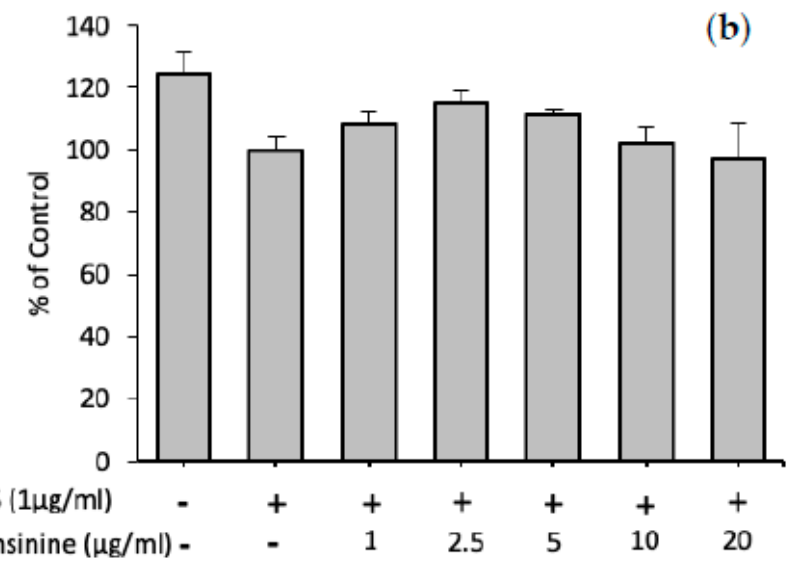

(b)

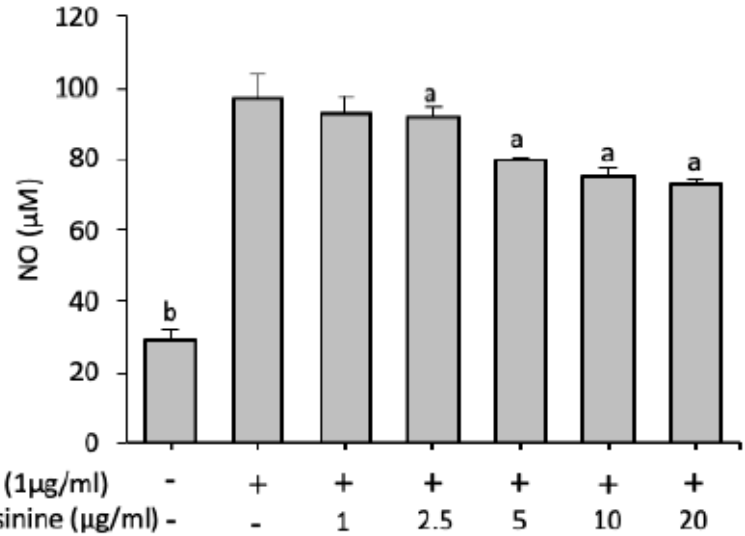

(c)

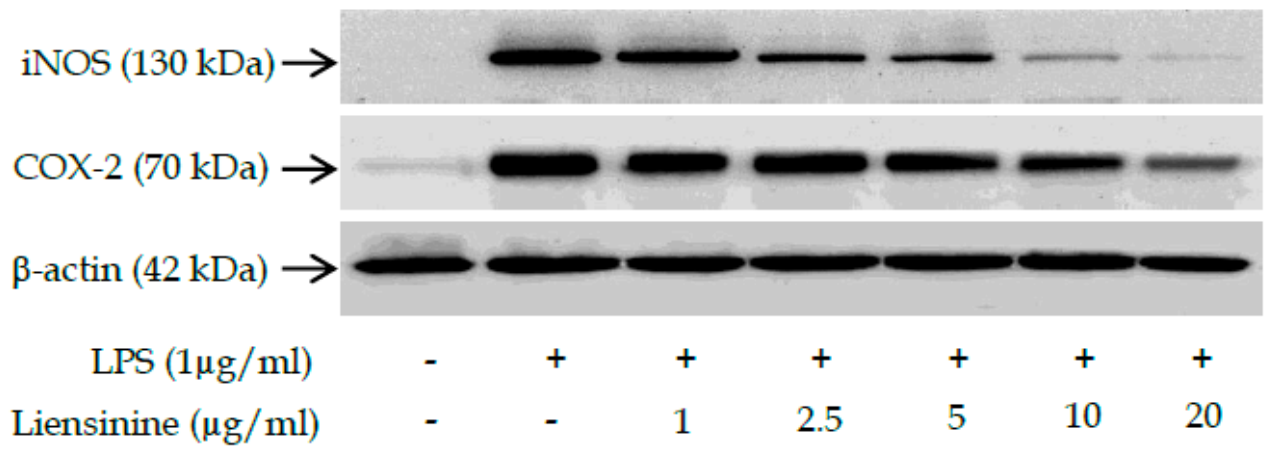

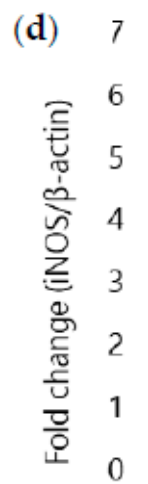

$\operatorname{LPS}(1 \mu \mathrm{g} / \mathrm{ml})$

Liensinine $(\mu \mathrm{g} / \mathrm{m})$
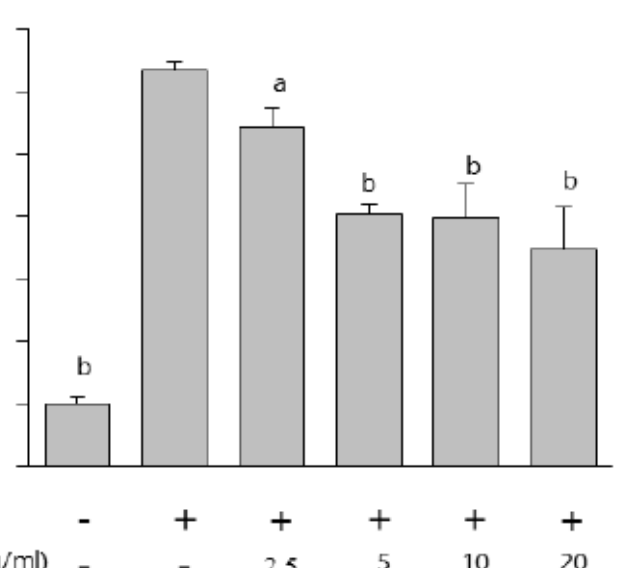

(e)

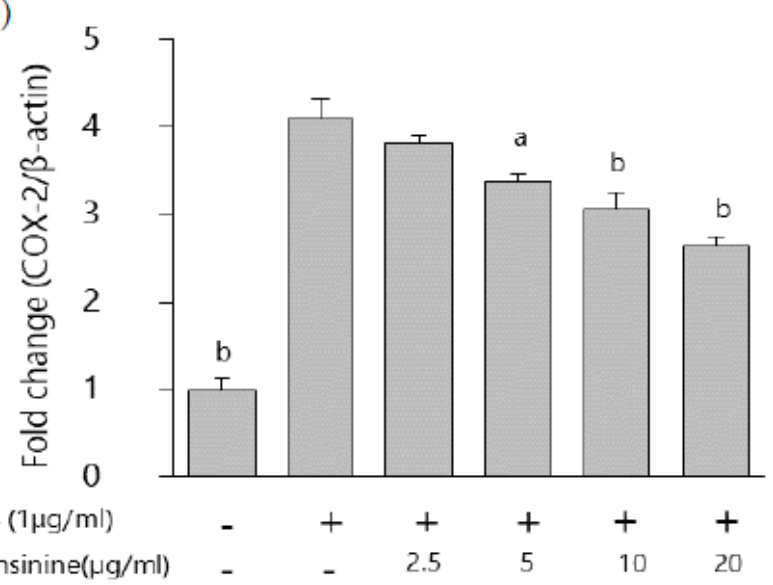

Figure 6. The effects of liensinine on cell viability, nitric oxide (NO) release, and protein expression of iNOS and COX-2. (a) Cytotoxicity/cell viability was done by MTT colorimetric assay. (b) The effect of liensinine on NO release was determined by Griess reagent assay. (c) The expression of the inflammatory proteins iNOS and COX-2 was done by immunoblot. $\beta$-actin was used as housekeeping/reference control to calculate relative fold change. (d) The fold change of iNOS. (e) The fold change of COX-2. ${ }^{\mathrm{a}} p<0.05$ and ${ }^{\mathrm{b}} p<0.01$ vs. Control (only LPS). $\mathrm{n}=3$ replicates. 


\section{Discussion}

In our study, we have shown that liensinine inhibits the key features of vascular inflammation mediated by altered VSMC function due to PDGF and TNF- $\alpha$, and macrophage function by LPS (Figure 7). During vascular inflammation, toxic insults to the blood vessel wall are mediated by oxidative stress, lipid peroxidation, and inflammation mediators released by VSMC and activated macrophage facilitates atherosclerosis progression [29,30]. Under stressful conditions, our bodies generate free radicals such as superoxide anion, which in turn convert NO to peroxynitrite. Peroxynitrite facilitates the oxidative modification of cholesterol to produce enormous quantities of lipid peroxidation byproducts [18]. Blood/serum lipids such as low-density lipoprotein (LDL) are involved in the progression/pathogenesis of numerous diseases including atherosclerosis. Oxidized-LDL upregulates the scavenger receptors on macrophages followed by the increased engulfment of ox-LDL and conversion of macrophages to foam cells characterized by accumulation of fatty streaks [31,32]. Therefore, pharmacological intervention inhibiting serum lipid peroxidation can slow down the process of vascular inflammation. We have previously shown that alkaloid rich fractions of Nelumbo nucifera possess strong antioxidant activity and suppress restenosis in a rat model [12]. As liensinine is one of the major alkaloids presents in Nelumbo nucifera, we sought to investigate if an antioxidant effect is exerted by liensinine. In our antioxidant activity assay, liensinine showed potent activity as revealed by scavenging the DPPH free radical with $\mathrm{IC}_{50}$ of $1.8 \mu \mathrm{g} / \mathrm{mL}$ (Figure 2a) and significantly inhibiting serum lipid peroxidation with 30 and $40 \mu \mathrm{g} / \mathrm{mL}$ concentrations of liensinine (Figure $2 \mathrm{~b}$ ). The DPPH antioxidant activity of liensinine was even better than another major alkaloid, neferine, with $\mathrm{IC}_{50}$ of $10.665 \mu \mathrm{g} / \mathrm{mL}(17.01 \mu \mathrm{M})$ [33].
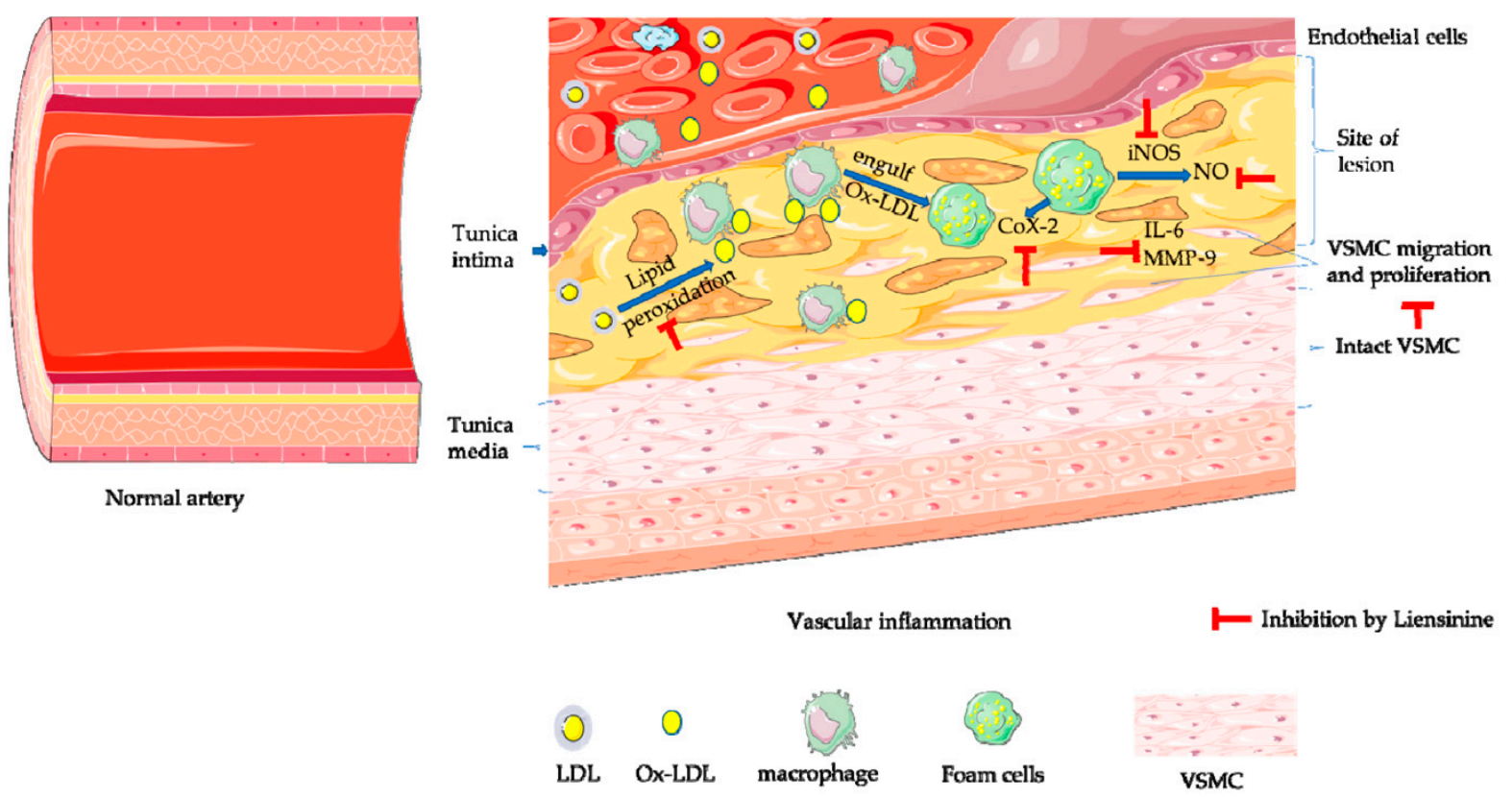

Figure 7. The mechanism of action of liensinine to inhibit vascular inflammation: During the progression of vascular inflammation, low density lipoprotein (LDL) is oxidized to ox-LDL by free radicals and these ox-LDL are engulfed/phagocytized by macrophages. The macrophage converts its phenotype to activated foam cells distinguished by the accumulation of fatty streaks of ox-LDL. These activated macrophages release numbers of inflammation mediators such as nitric oxide (NO), TNF$\alpha$, inducible nitric oxide synthase (iNOS) and cyclooxygenase (COX-2). Similarly, the intact vascular smooth cells (VSMC) in the tunica media are activated by cytokines such as TNF- $\alpha$ and growth factor such as PDGF. VSMCs release cytokines such as IL-6 and initiate proliferation mediated by PDGF and migration mediated by matrix metalloproteinase-9 (MMP-9) enzymatic activity at the site of a lesion. Collectively, this process leads to vascular inflammation flowed by atherosclerosis. 
In physiological systems, atherogenesis is initiated in major arteries after endothelial dysfunction triggered by oxidative stress, leading to significant changes in the permeability of the vascular intimal layer and resulting in transportation of ox-LDL to the vascular inner layer [34]. After endothelial cells are activated by atherogenic risk factors such as ox-LDL, they overexpress cell adhesion molecules such as intercellular adhesion molecules and vascular cell adhesion molecules to attract circulating cells including monocytes and leukocytes. The transported ox-LDL are engulfed by scavenger receptors of macrophages/monocytes [35]. Macrophages are activated after phagocytosis of ox-LDL and in turn overexpress iNOS and COX-2 [17,36]. It is well established that high iNOS level corresponds with a massive release of $\mathrm{NO}$ from macrophages and subsequent inflammatory response [37]. Similarly, LDL also stimulates the production of various prostaglandins through the COX-2 pathway, and these prostaglandins are known to be mitogenic, simulating cell proliferation [38]. Our results showed promising activity of liensinine to suppress NO release from LPS-induced RAW264.7. The $25 \%$ reduction of NO by liensinine at a concentration of $20 \mu \mathrm{g} / \mathrm{mL}$ (Figure $6 \mathrm{~b}$ ) was comparable to $20 \mu \mathrm{M}$ of neferine [33]. Furthermore, liensinine also notably decreased the protein expression of COX-2 and iNOS. The trend of iNOS and COX-2 inhibition shown by liensinine was similar to that shown by glucosamine (a commercially available anti-inflammatory drug) at a concentration of 2.5 to $10 \mathrm{mM}$ in LPS-induced RAW264.7 cells [39].

IL-6 is a well characterized inflammatory mediator and it is released by VSMC after the induction of potent stimulants such as TNF- $\alpha$ [40] and angiotensin II [41]. IL-6 is crucial in vascular remodelling and it, along with ox-LDL, is a potential prognostic marker in predicting cerebral vascular and cardiovascular disorders [42]. In our ELISA result (Figure 5), liensinine suppressed the TNF- $\alpha$ induced IL- 6 level in VSMC by approximately $50 \%$ at $30 \mu \mathrm{g} / \mathrm{mL}$ concentration. During atherosclerosis, the vascular lumen is narrowed by a fibrous cap composed of (among many other things) VSMC and extracellular matrix. Various growth factors and cytokines produced by endothelial cells and inflammatory cells contribute to the proliferation and migration of VSMC leading to fibrous cap formation [29,43,44]. Cytokines like PDGF can induce proliferation of VSMC to a significantly high level, whereas TNF- $\alpha$ is known to stimulate the migration of VSMC from tunica media to the site of a lesion by increasing MMP-9 expression. MMP-9 is a key gelatinolytic enzyme responsible for the degradation of the elastic lamina barrier of the extra cellular matrix $[45,46]$. In our previous publications, we have shown that PDGF and TNF- $\alpha$ promote the proliferation and MMP-9-dependent migration of VSMC $[11,12,14]$. In our result, liensinine inhibited the PDGF-BB induced proliferation/growth of VSMC, revealing its potent anti-proliferating activity. At a $30 \mu \mathrm{g} / \mathrm{mL}$ concentration of liensinine, the proliferation of VSMC was almost completely inhibited to the level of the control (without PDGF) (Figure 3). The potent anti-proliferative activity of liensinine at a concentration of $30 \mu \mathrm{g} / \mathrm{mL}$ is comparable to $50 \mu \mathrm{M}$ of epigallocatechin3-O-gallate [47] and 20mM of carnosine [48]. Likewise, liensinine at a concentration of 10,20 and $30 \mu \mathrm{g} / \mathrm{mL}$ significantly attenuated the expression of MMP-9 induced by TNF- $\alpha$ (Figure 4). We speculate that the notable inhibition of MMP-9 enzymatic activity by Nelumbo nucifera leaf extract in our previous study, at a concentration of $250 \mu \mathrm{g} / \mathrm{mL}$, was in part exerted by liensinine [23]. Taken together, our results provide the mechanistic pathway to attenuate the progression of atherogenesis via controlling vascular inflammation by liensinine possibly by targeting VSMC proliferation, MMP-9 expression and inflammatory mediators released by macrophages (Figure 7).

\section{Conclusions}

Our results showed that liensinine can effectively prevent the progression of atherosclerosis by modulating the mediators of vascular inflammation via inhibiting the migration and proliferation of VSMC as well as attenuating the release of inflammatory mediators from RAW264.7. Our research is limited to an in vitro experiment, therefore further research is necessary to explore the protective role of liensinine in the pathophysiology of 
atherosclerosis. It would be worth conducting an in vivo study in a pre-clinical animal model of atherosclerosis and exploring the promising activity of liensinine.

Author Contributions: Conceptualization, D.-W.K. and M.Y.J.; methodology, R.K. and K.R.P.; software, M.Y.J. and R.K.; investigation, M.Y.J. and R.K.; writing—original draft preparation, R.K., K.R.P.; writing-R.K. and K.R.P.; review and editing, N.P. and H.P.D.; supervision, D.-W.K. All authors have read and agreed to the published version of the manuscript.

Funding: This research received no external funding.

Institutional Review Board Statement: Not applicable.

Informed Consent Statement: Not applicable.

Data Availability Statement: The data presented in this study are available on request from the corresponding author.

Acknowledgments: The authors would like to acknowledge the research support provided by Mokpo National University, Muan-gun, Jeonnam, Korea.

Conflicts of Interest: The authors declare no conflict of interest.

\section{References}

1. Stemme, S.; Faber, B.; Holm, J.; Wiklund, O.; Witztum, J.L.; Hansson, G.K. T lymphocytes from human atherosclerotic plaques recognize oxidized low density lipoprotein. Proc. Natl. Acad. Sci. USA 1995, 92, 3893-3897. [CrossRef] [PubMed]

2. Libby, P.; Ridker, P.M.; Maseri, A. Inflammation and atherosclerosis. Circulation 2002, 105, 1135-1143. [CrossRef] [PubMed]

3. Choi, K.H.; Kim, J.E.; Song, N.R.; Son, J.E.; Hwang, M.K.; Byun, S.; Kim, J.H.; Lee, K.W.; Lee, H.J. Phosphoinositide 3-kinase is a novel target of piceatannol for inhibiting PDGF-BB-induced proliferation and migration in human aortic smooth muscle cells. Cardiovasc. Res. 2010, 85, 836-844. [CrossRef] [PubMed]

4. Paudel, K.R.; Kim, D.W. Microparticles-Mediated Vascular Inflammation and its Amelioration by Antioxidant Activity of Baicalin. Antioxidants 2020, 9, 890. [CrossRef] [PubMed]

5. Huber, S.A.; Sakkinen, P.; Conze, D.; Hardin, N.; Tracy, R. Interleukin-6 exacerbates early atherosclerosis in mice. Arterioscler. Thromb. Vasc. Biol. 1999, 19, 2364-2367. [CrossRef]

6. Moon, S.K.; Cha, B.Y.; Kim, C.H. ERK1/2 mediates TNF-alpha-induced matrix metalloproteinase-9 expression in human vascular smooth muscle cells via the regulation of NF-kappaB and AP-1: Involvement of the ras dependent pathway. J. Cell Physiol. 2004, 198, 417-427. [CrossRef]

7. Zhang, X.; Hu, W.; Wu, F.; Yuan, X.; Hu, J. Shikonin inhibits TNF-alpha-induced growth and invasion of rat aortic vascular smooth muscle cells. Can. J. Physiol. Pharmacol. 2015, 93, 615-624. [CrossRef]

8. Yang, G.; Zhou, X.; Chen, T.; Deng, Y.; Yu, D.; Pan, S.; Song, Y. Hydroxysafflor yellow A inhibits lipopolysaccharide-induced proliferation and migration of vascular smooth muscle cells via Toll-like receptor-4 pathway. Int. J. Clin. Exp. Med. 2015, $8,5295-5302$.

9. Osman, I.; Segar, L. Pioglitazone, a PPARgamma agonist, attenuates PDGF-induced vascular smooth muscle cell proliferation through AMPK-dependent and AMPK-independent inhibition of mTOR/p70S6K and ERK signaling. Biochem. Pharmacol. 2016, 101, 54-70. [CrossRef]

10. Paudel, K.R.; Oak, M.H.; Kim, D.W. Smooth Muscle Cell Derived Microparticles Acts as Autocrine Activation of Smooth Muscle Cell Proliferation by Mitogen Associated Protein Kinase Upregulation. J. Nanosci. Nanotechnol. 2020, 20, 5746-5750. [CrossRef]

11. Lee, H.H.; Paudel, K.R.; Kim, D.W. Terminalia chebula Fructus Inhibits Migration and Proliferation of Vascular Smooth Muscle Cells and Production of Inflammatory Mediators in RAW 264.7. Evid. Based Complement. Altern. Med. 2015, $2015,502182$. [CrossRef] [PubMed]

12. Jun, M.Y.; Karki, R.; Paudel, K.R.; Sharma, B.R.; Adhikari, D.; Kim, D.W. Alkaloid rich fraction from Nelumbo nucifera targets VSMC proliferation and migration to suppress restenosis in balloon-injured rat carotid artery. Atherosclerosis 2016, 248, 179-189. [CrossRef] [PubMed]

13. Whatling, C.; McPheat, W.; Hurt-Camejo, E. Matrix management: Assigning different roles for MMP-2 and MMP-9 in vascular remodeling. Arterioscler. Thromb. Vasc. Biol. 2004, 24, 10-11. [CrossRef] [PubMed]

14. Paudel, K.R.; Karki, R.; Kim, D.W. Cepharanthine inhibits in vitro VSMC proliferation and migration and vascular inflammatory responses mediated by RAW264.7. Toxicol. In Vitro 2016, 34, 16-25. [CrossRef]

15. Maiolino, G.; Rossitto, G.; Caielli, P.; Bisogni, V.; Rossi, G.P.; Calo, L.A. The role of oxidized low-density lipoproteins in atherosclerosis: The myths and the facts. Mediat. Inflamm. 2013, 2013, 714653. [CrossRef]

16. Lee, H.H.; Paudel, K.R.; Jeong, J.; Wi, A.J.; Park, W.S.; Kim, D.W.; Oak, M.H. Antiatherogenic Effect of Camellia japonica Fruit Extract in High Fat Diet-Fed Rats. Evid. Based Complement. Altern. Med. 2016, 2016, 9679867. [CrossRef] 
17. Baker, C.S.; Hall, R.J.; Evans, T.J.; Pomerance, A.; Maclouf, J.; Creminon, C.; Yacoub, M.H.; Polak, J.M. Cyclooxygenase-2 is widely expressed in atherosclerotic lesions affecting native and transplanted human coronary arteries and colocalizes with inducible nitric oxide synthase and nitrotyrosine particularly in macrophages. Arterioscler. Thromb. Vasc. Biol. 1999, 19, 646-655. [CrossRef]

18. Panth, N.; Paudel, K.R.; Parajuli, K. Reactive Oxygen Species: A Key Hallmark of Cardiovascular Disease. Adv. Med. 2016, 2016, 9152732. [CrossRef]

19. Meng, X.-L.; Chen, M.-L.; Chen, C.-L.; Gao, C.-C.; Li, C.; Wang, D.; Liu, H.-S.; Xu, C.-B. Bisbenzylisoquinoline alkaloids of lotus (Nelumbo nucifera Gaertn.) seed embryo inhibit lipopolysaccharide-induced macrophage activation via suppression of $\mathrm{Ca}^{2+}-\mathrm{CaM} / \mathrm{CaMKII}$ pathway. Food Agric. Immunol. 2019, 30, 878-896. [CrossRef]

20. Meng, X.-L.; Zheng, L.-C.; Liu, J.; Gao, C.-C.; Qiu, M.-C.; Liu, Y.-Y.; Lu, J.; Wang, D.; Chen, C.-L. Inhibitory effects of three bisbenzylisoquinoline alkaloids on lipopolysaccharide-induced microglial activation. RSC Adv. 2017, 7, 18347-18357. [CrossRef]

21. Sharma, B.R.; Gautam, L.N.; Adhikari, D.; Karki, R. A Comprehensive Review on Chemical Profiling of Nelumbo Nucifera: Potential for Drug Development. Phytother. Res. 2017, 31, 3-26. [CrossRef] [PubMed]

22. Paudel, K.R.; Panth, N. Phytochemical Profile and Biological Activity of Nelumbo nucifera. Evid. Based Complement. Altern. Med. 2015, 2015, 789124. [CrossRef] [PubMed]

23. Karki, R.; Jeon, E.R.; Kim, D.W. Nelumbo nucifera leaf extract inhibits neointimal hyperplasia through modulation of smooth muscle cell proliferation and migration. Nutrition 2013, 29, 268-275. [CrossRef] [PubMed]

24. Panth, N.; Paudel, K.R.; Gong, D.S.; Oak, M.H. Vascular Protection by Ethanol Extract of Morus alba Root Bark: EndotheliumDependent Relaxation of Rat Aorta and Decrease of Smooth Muscle Cell Migration and Proliferation. Evid. Based Complement. Altern. Med. 2018, 2018, 7905763. [CrossRef] [PubMed]

25. Paudel, K.R.; Wadhwa, R.; Mehta, M.; Chellappan, D.K.; Hansbro, P.M.; Dua, K. Rutin loaded liquid crystalline nanoparticles inhibit lipopolysaccharide induced oxidative stress and apoptosis in bronchial epithelial cells in vitro. Toxicol. In Vitro 2020, 68, 104961. [CrossRef] [PubMed]

26. Kim, T.M.; Paudel, K.R.; Kim, D.W. Eriobotrya japonica leaf extract attenuates airway inflammation in ovalbumin-induced mice model of asthma. J. Ethnopharmacol. 2020, 253, 112082. [CrossRef]

27. Tanizawa, S.; Ueda, M.; van der Loos, C.M.; van der Wal, A.C.; Becker, A.E. Expression of platelet derived growth factor B chain and beta receptor in human coronary arteries after percutaneous transluminal coronary angioplasty: An immunohistochemical study. Heart 1996, 75, 549-556. [CrossRef]

28. Karki, R.; Jeon, E.R.; Kim, D.W. Magnoliae Cortex inhibits intimal thickening of carotid artery through modulation of proliferation and migration of vascular smooth muscle cells. Food Chem. Toxicol. 2012, 50, 634-640. [CrossRef]

29. Ross, R. Atherosclerosis-An inflammatory disease. N. Engl. J. Med. 1999, 340, 115-126. [CrossRef]

30. Paudel, K.R.; Panth, N.; Kim, D.W. Circulating Endothelial Microparticles: A Key Hallmark of Atherosclerosis Progression. Scientifica 2016, 2016, 8514056. [CrossRef]

31. Paudel, K.R.; Lee, U.W.; Kim, D.W. Chungtaejeon, a Korean fermented tea, prevents the risk of atherosclerosis in rats fed a high-fat atherogenic diet. J. Integr. Med. 2016, 14, 134-142. [CrossRef]

32. Steinberg, D. Role of oxidized LDL and antioxidants in atherosclerosis. Adv. Exp. Med. Biol. 1995, 369, 39-48. [CrossRef] [PubMed]

33. Jung, H.A.; Jin, S.E.; Choi, R.J.; Kim, D.H.; Kim, Y.S.; Ryu, J.H.; Kim, D.W.; Son, Y.K.; Park, J.J.; Choi, J.S. Anti-amnesic activity of neferine with antioxidant and anti-inflammatory capacities, as well as inhibition of ChEs and BACE1. Life Sci. 2010, 87, 420-430. [CrossRef] [PubMed]

34. Tabas, I.; Williams, K.J.; Boren, J. Subendothelial lipoprotein retention as the initiating process in atherosclerosis: Update and therapeutic implications. Circulation 2007, 116, 1832-1844. [CrossRef]

35. Stephen, S.L.; Freestone, K.; Dunn, S.; Twigg, M.W.; Homer-Vanniasinkam, S.; Walker, J.H.; Wheatcroft, S.B.; Ponnambalam, S. Scavenger receptors and their potential as therapeutic targets in the treatment of cardiovascular disease. Int. J. Hypertens. 2010, 2010, 646929. [CrossRef]

36. Karki, R.; Park, C.H.; Kim, D.W. Extract of buckwheat sprouts scavenges oxidation and inhibits pro-inflammatory mediators in lipopolysaccharide-stimulated macrophages (RAW264.7). J. Integr. Med. 2013, 11, 246-252. [CrossRef]

37. Crow, J.P.; Beckman, J.S. Reactions between nitric oxide, superoxide, and peroxynitrite: Footprints of peroxynitrite in vivo. Adv. Pharmacol. 1995, 34, 17-43. [CrossRef]

38. Kreuzer, J.; Denger, S.; Jahn, L.; Bader, J.; Ritter, K.; von Hodenberg, E.; Kubler, W. LDL stimulates chemotaxis of human monocytes through a cyclooxygenase-dependent pathway. Arterioscler. Thromb. Vasc. Biol. 1996, 16, 1481-1487. [CrossRef]

39. Rafi, M.M.; Yadav, P.N.; Rossi, A.O. Glucosamine inhibits LPS-induced COX-2 and iNOS expression in mouse macrophage cells (RAW 264.7) by inhibition of p38-MAP kinase and transcription factor NF-kappaB. Mol. Nutr. Food Res. 2007, 51, 587-593. [CrossRef]

40. Wang, Z.; Castresana, M.R.; Newman, W.H. NF-kappaB is required for TNF-alpha-directed smooth muscle cell migration. FEBS Lett. 2001, 508, 360-364. [CrossRef]

41. Funakoshi, Y.; Ichiki, T.; Ito, K.; Takeshita, A. Induction of interleukin-6 expression by angiotensin II in rat vascular smooth muscle cells. Hypertension 1999, 34, 118-125. [CrossRef] [PubMed]

42. Lobbes, M.B.; Lutgens, E.; Heeneman, S.; Cleutjens, K.B.; Kooi, M.E.; van Engelshoven, J.M.; Daemen, M.J.; Nelemans, P.J. Is there more than C-reactive protein and fibrinogen? The prognostic value of soluble CD40 ligand, interleukin-6 and oxidized low-density lipoprotein with respect to coronary and cerebral vascular disease. Atherosclerosis 2006, 187, 18-25. [CrossRef] 
43. Lusis, A.J. Atherosclerosis. Nature 2000, 407, 233-241. [CrossRef] [PubMed]

44. Libby, P.; Okamoto, Y.; Rocha, V.Z.; Folco, E. Inflammation in atherosclerosis: Transition from theory to practice. Circ. J. 2010, 74, 213-220. [CrossRef]

45. Karki, R.; Ho, O.M.; Kim, D.W. Magnolol attenuates neointima formation by inducing cell cycle arrest via inhibition of ERK1/2 and NF-kappaB activation in vascular smooth muscle cells. Biochim. Biophys. Acta 2013, 1830, 2619-2628. [CrossRef] [PubMed]

46. Karki, R.; Kim, S.B.; Kim, D.W. Magnolol inhibits migration of vascular smooth muscle cells via cytoskeletal remodeling pathway to attenuate neointima formation. Exp. Cell Res. 2013, 319, 3238-3250. [CrossRef]

47. Lee, M.H.; Kwon, B.J.; Koo, M.A.; You, K.E.; Park, J.C. Mitogenesis of vascular smooth muscle cell stimulated by platelet-derived growth factor-bb is inhibited by blocking of intracellular signaling by epigallocatechin-3-O-gallate. Oxid. Med. Cell. Longev. 2013, 2013, 827905. [CrossRef]

48. Hwang, B.; Song, J.H.; Park, S.L.; Kim, J.T.; Kim, W.J.; Moon, S.K. Carnosine Impedes PDGF-Stimulated Proliferation and Migration of Vascular Smooth Muscle Cells In Vitro and Sprout Outgrowth Ex Vivo. Nutrients 2020, 12, 2697. [CrossRef] 